\title{
The development of GAMA Swab sampling chamber for walk-through sampling in patients with COVID-19 at Gadjah Mada Hospital
}

\author{
Hera Nirwati, ${ }^{1 *}$ Dwi Aris Agung Nugrahaningsih, ${ }^{2}$ Siswanto, ${ }^{3}$ Mahatma Sotya Bawono, ${ }^{3}$ \\ Titien Budhiaty, ${ }^{3}$ Setyawan Bekti Wibowo, ${ }^{4}$ Raden Sumiharto ${ }^{5}$
}

\author{
'Department of Microbiology, Faculty of Medicine, Public Health and Nursing, Universitas Gadjah Mada, Yogyakarta, \\ Indonesia \\ ${ }^{2}$ Department of Pharmacology and Therapy, Faculty of Medicine, Public Health and Nursing, Universitas Gadjah Mada, \\ Yogyakarta, Indonesia \\ ${ }^{3}$ Universitas Gadjah Mada Academic Hospital, Yogyakarta, Indonesia \\ ${ }^{4}$ Department of Mechanical Engineering, Vocational School, Universitas Gadjah Mada, Yogyakarta, Indonesia \\ ${ }^{5}$ Department of Computer Science and Electronics, Faculty of Mathematics and Natural Sciences, Universitas Gadjah \\ Mada, Yogyakarta, Indonesia
}

\section{KEYWORDS}

Chamber testing COVID-19

Swab sampling Walk-through

\begin{abstract}
Collecting swab samples from the nasopharynx and oropharynx of patients with COVID-19 is essential in detecting SARS-CoV-2. This procedure potentially produces sufficient droplets. Since SARS-CoV-2 is transmitted through droplets, swab sampling has to be done carefully to prevent the risk of transmission to healthcare workers or the cross-contamination to the environment. The GAMA Swab Sampling Chamber (GSSC) is a positive-pressure chamber designed for collecting swab samples involving the healthcare worker positioned inside, while the patient is outside the chamber. The chamber is designed to minimize the risk of aerosol exposure to the healthcare worker due to leakage or when opening or closing the door. Accordingly, the healthcare worker does not need to use complete personal protective equipment (PPE) as they do when collecting swab samples without the chamber. After several tests to check the safety and the chamber's function, the GSSC was used at Gadjah Mada Hospital. This chamber had been used to swab 51 asymptomatic patients, 72 suspected patients, and 284 voluntary persons for ten weeks. The results of reverse transcription-polymerase chain reaction (RT-PCR) examination of all samples from asymptomatic patients were negative, while 2 of 72 suspected patients $(2.8 \%)$ and 4 of 284 voluntary persons (1.4\%) had positive RT-PCR results. The use of GSSC can simplify the swab sampling, also reduces the need for PPE usage and a negative pressure isolation room which are limited in the current pandemic situation.
\end{abstract}

(c) The Journal 2020. This article is distributed under a Creative Commons Attribution-ShareAlike 4.0 International license.

\section{Introduction}

On March 12, 2020, The World Health Organization (WHO) declared the Coronavirus Disease 19 (COVID-19) as a new global pandemic in the world. COVID-19 is caused by Severe Acute Respiratory Syndrome Coronavirus 2 (SARS-CoV-2). ${ }^{1}$ This virus has quickly spread throughout the world. By July 2 , 2020, 215 countries and territories reported having patients with COVID-19, with a total of $10,829,468$

*Correspondence: hera.nirwati@mail.ugm.ac.id Department of Microbiology, Faculty of Medicine, Public Health, and Nursing, Universitas Gadjah Mada, Yogyakarta, Jl. Farmako, Sekip Utara, Yogyakarta 55281, Indonesia cases. Of these cases, 6,048,749 people were reported recovered, and 519,397 of them died. Presently, the United States, Brazil, and Russia are the top three countries with the largest number of COVID-19 cases in the world. ${ }^{2}$

In Indonesia, COVID-19 was first reported on March 2, 2020, in Jakarta. Since then, the virus has spread quickly throughout the country. July 2, 2020, there were 59,394 cases of COVID-9, with 26,667 cases recovered and 2,987 deaths. In Yogyakarta, 320 confirmed cases were reported, with 269 cases recovered, and eight deaths. ${ }^{3}$

One effective strategy in managing COVID-19 
is test, track, and trace. Real-time reverse transcriptase-polymerase chain reaction (RT-PCR) results of nasopharyngeal and oropharyngeal swabs typically have been used to confirm the clinical diagnosis. ${ }^{4}$ With an increasing number of suspected and symptomatic individuals to be tested, there is a need for a safe and efficient screening system. The process also needs a large laboratory capacity for RTPCR testing. To overcome this problem, the Indonesia government has increased the capacity of RT-PCR testing. As of July 2, 2020, RT-PCR tests have been conducted on 849,155 samples, with 59,394 people who tested positive. ${ }^{3}$

Collecting nasopharyngeal and oropharyngeal swabs from patients with COVID-19 is a procedure that potentially produces sufficient droplets for accurate testing results. Since SARS-CoV-2 is transmitted through droplets, swab sampling has to be done carefully to prevent the risk of transmission to healthcare workers or the cross-contamination to the environment. The healthcare worker must use personal protective equipment (PPE) to protect themselves. This procedure also has to be done in a negative pressure room to prevent environmental contamination.

The sampling process often faces the obstacles of limited PPE stocks. Also, the availability of a negative pressure room is limited because not all hospitals and laboratories have one. In response to this problem, the research team developed the GAMA Swab Sampling Chamber to be used for a safe and efficient swab sampling system.

\section{Method}

\subsection{Development of the GAMA Swab Sampling Chamber}

The GAMA Swab Sampling Chamber (GSSC) was a positive pressure chamber designed for collecting nasopharyngeal and oropharyngeal swabs from the patients with COVID-19 with the healthcare worker positioned inside while the patient was outside the chamber. The positive pressure chamber was designed to minimalize the risk of aerosol exposure to the healthcare worker due to leakage or when opening or closing the door.

The size of the chamber was $1.2 \mathrm{~m} \times 1.2 \mathrm{~m} \times 1.2 \mathrm{~m}$ and wide enough to be used by healthcare workers of various sizes. The chamber was mobile and designed to be easily moved using the attached wheels. To make a strong construction but light in weight, the chamber was built using rust-resistant aluminium material with a $5 \mathrm{~mm}$ acrylic wall. The chamber was designed with transparent walls, one of which had a pair of long gloves for a healthcare worker to use to take the nasopharyngeal and oropharyngeal swabs for COVID-19 testing using the RT-PCR method.

The safety aspect of the healthcare worker and the environment was a priority when developing this chamber. In addition to being pressurized positively, this chamber was equipped with a HEPA filter to keep the air circulation and exhaust system safer. The HEPA H14 filter, which has $99.995 \%$ efficiency in filtering material less than 0.3 microns, was chosen. ${ }^{5}$

Additionally, the chamber was equipped with an air cooler to make the healthcare worker comfortable inside the chamber. There was a microphone attached to the acrylic plexi-glass wall to communicate with the patient or person outside the chamber. There was also a spotlight for lighting. The construction was designed to have a limited indentation in order to be cleaned easily. The chamber had a disinfection system with a UV light and $\mathrm{H}_{2} \mathrm{O}_{2}$ dry misting system. The dry misting system was used to disinfect the surface of the chamber facing the patient's side during patient turnover. The UV light was used to sterilize the entire chamber after use. The construction scheme of the chamber is described in Figure 1.

\subsection{GAMA Swab Sampling Chamber testing}

Some testing was done to ensure that the chamber can be operated following the medical equipment standardization. Construction and airflow testing using either numerical simulations or experimental testing had been done. We examined the air capacity that passes through and the pressure difference that occurred between inside and outside the chamber (Figure 2). Initial testing was conducted using the Computational Fluid Dynamics (CFD) technique simulation to estimate the capacity requirements and size of the air ducts according to the standards. Simulations were done at the same time to see the direction of the airflow and pressure distribution with the simulation configuration using the eddy viscosity 

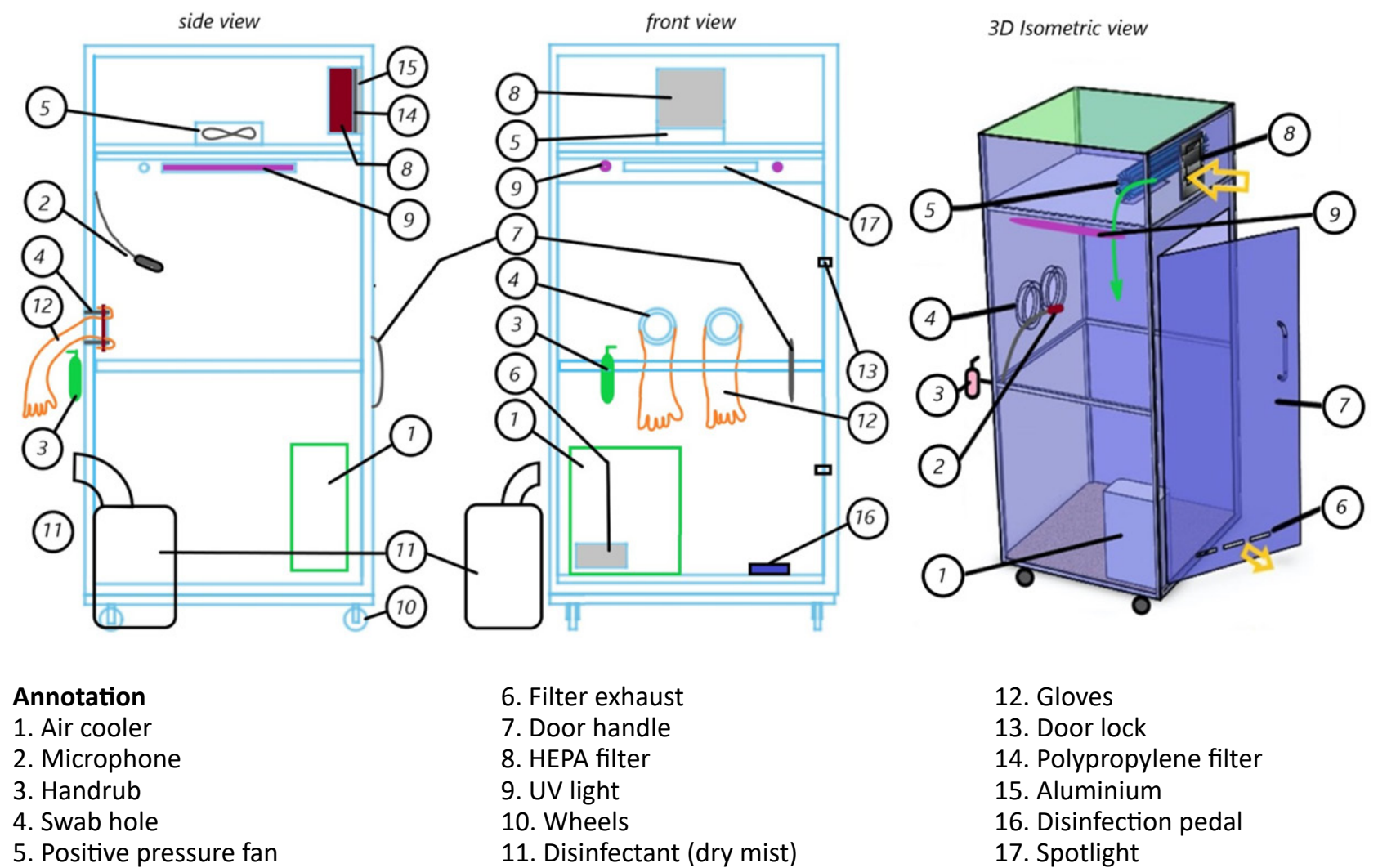
6. Filter exhaust
7. Door handle
8. HEPA filter
9. UV light
10. Wheels
11. Disinfectant (dry mist)

\begin{abstract}
12. Gloves
13. Door lock

14. Polypropylene filter

15. Aluminium

16. Disinfection pedal

17. Spotlight
\end{abstract}

Figure 1. GAMA Swab Sampling Chamber construction scheme

$k-\varepsilon$ turbulence equation. For the experimental testing, measurements were made for different pressure conditions using a magnehelic gauge and impermeability testing by using fogging smoke inside the chamber.

There were also some tests to check the cubicle's tightness and the function of the chamber. The cubicle's tightness was tested by observing the smoke flow from inside the chamber. The functional tests were done by clinicians actually swabbing the simulated patients.

\subsection{Location of GAMA Swab Sampling Chamber}

The GAMA Swab Sampling Chamber is dedicated to walk-through outpatients sampling. In order to be utilized optimally, the chamber is located in an open space outside the Emergency Department of Gadjah Mada Hospital. Because it is located outdoors, the patient queue can be arranged with a safe distance. Sunlight exposure helps in ensuring the remnants of aerosols can be disinfected by UV in the sunlight to minimize the cross-contamination to the environment.

\subsection{Swab sampling}

The procedure that the patient must follow for taking nasopharyngeal and oropharyngeal swabs: (1) register at Gadjah Mada Hospital, (2) fill in the patient registration link, (3) get a queue schedule, (4) follow the sampling instructions in the GAMA Swab Sampling Chamber, and (5) get the results via email or WhatsApp. Patients with positive RT-PCR results will be informed and referred for further examination at the Respiratory Clinic of Gadjah Mada Hospital.

\subsection{The usage of the GAMA Swab Sampling Chamber}

The GAMA Swab Sampling Chamber was used first on April 24, 2020. The data of patients using this chamber were documented in the hospital register. Then, the nasopharyngeal and oropharyngeal swabs were sent to Microbiology Laboratory, Faculty of Medicine, Public Health 


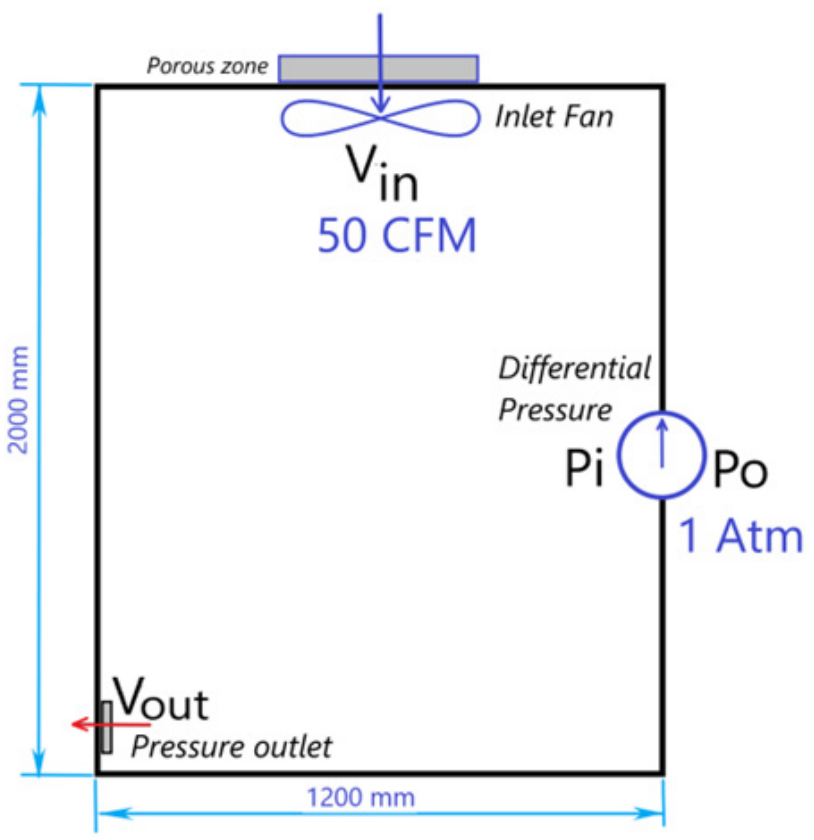

Figure 2. GAMA Swab Sampling Chamber testing scheme

and Nursing, Universitas Gadjah Mada for RTPCR testing. The results of the RT-PCR tests were documented in the register in the Clinical Pathology Laboratory of Gadjah Mada Hospital.

\subsection{Ethical approval}

The Medical and Health Research Ethics Committee, Faculty of Medicine, Public Health and Nursing, Universitas Gadjah Mada, Yogyakarta, Indonesia approved this study with letter number KE/FK/0680/EC/2020.

\section{Result}

The GAMA Swab Sampling Chamber was built in eSVe FabLab Vocational School Universitas Gadjah Mada with aluminium for the frame and $5 \mathrm{~mm}$ acrylic for the walls. The chamber was designed with transparent walls, one of which had a pair of long gloves for a healthcare worker to use to make nasopharyngeal and oropharyngeal swabs. This chamber was equipped with a HEPA filter, microphone, spotlight, water cooler, UV, and dry mist disinfectant (Figure 3a).

The results of the flow simulation, showing airflow's source through the HEPA filter at the top, then lead and was concentrated at the bottom of the chamber (Figure 3b). The effect of using a HEPA filter caused the airflow capacity to be 50 cubic feet per minute (CFM) so that the clean air exchange capacity reached 23 air changes per hour (ACH). Meanwhile, the effect of the flow entering the chamber caused the pressure in the chamber to be higher. The results of the pressure distribution showed that there was an average pressure difference of 12.13 $\mathrm{Pa}$, which was consistent with experimental testing using magnehelic differential pressure gauges. The measurement results showed a pressure difference in the chamber and outside air of around $12 \mathrm{~Pa}$ (Figure 3c).

The cubicle's tightness was tested by observing the smoke flow from inside the chamber (Figure 3d). Observations show that the smoke flows only through the outlet channel area. Meanwhile, functional tests were conducted by swab-collecting clinicians, and the results showed that the equipment inside the chamber was easy to use and comfortable, as shown in Figure 3e.

Swab sampling service with this chamber at Gadjah Mada Hospital has been conducted starting on April 24, 2020. The nasopharyngeal and oropharyngeal swabs are taken from asymptomatic patients, suspected patients, and voluntary persons in outdoor area in front of Emergency Department Gadjah Mada Hospital (Figure 4). Until July 3, 2020, swab sampling has been done on 407 patients, consisting of 51 asymptomatic patients, 72 suspected patients, and 284 voluntary persons. The results of RT-PCR showed 4 of 284 voluntary persons (1.4\%) and 2 of 72 suspected patients (2.8\%) had positive RT-PCR results, while all asymptomatic patients had negative results (Table 1).

\section{Discussion}

In the process of oropharyngeal and nasopharyngeal swabs collection, a limited number of patients were diagnosed due to the considerable time required for wearing and removing PPE and environmental decontamination. For this reason, several types of screening centers have been developed to enable rapid swabs collection. The prompt screening and swabs collection are essential at 


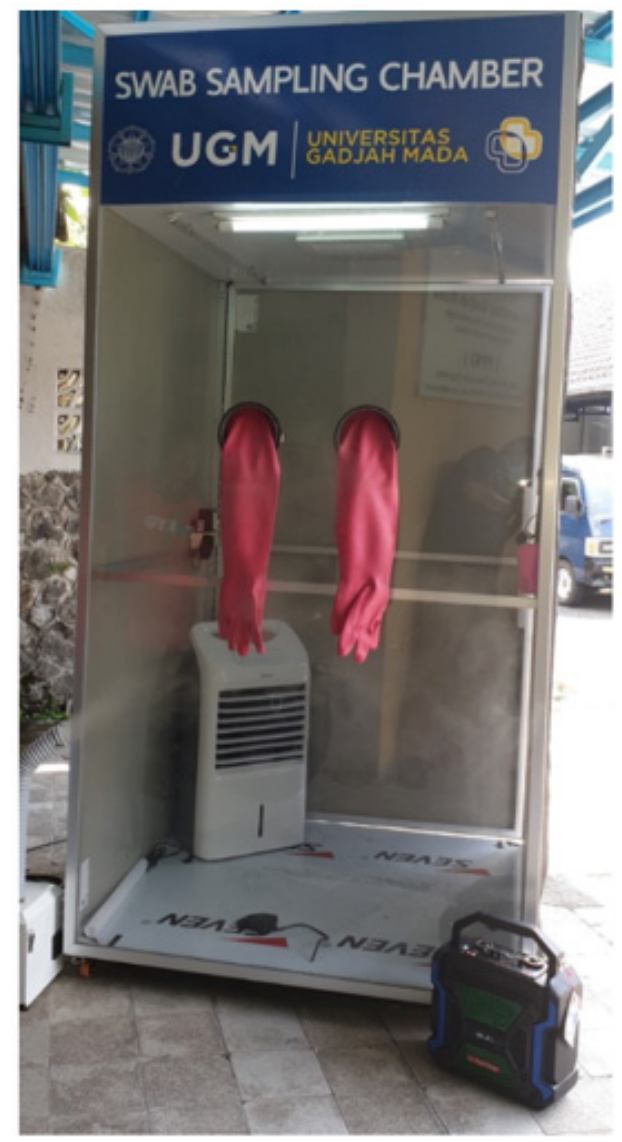

(a)

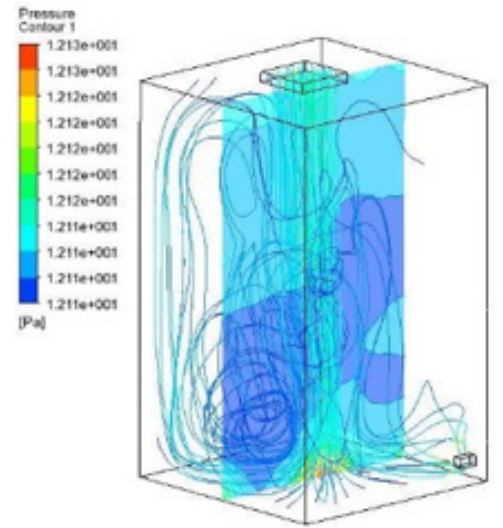

(b)

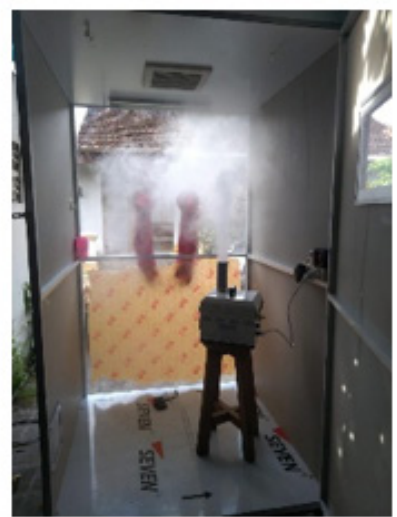

(d)

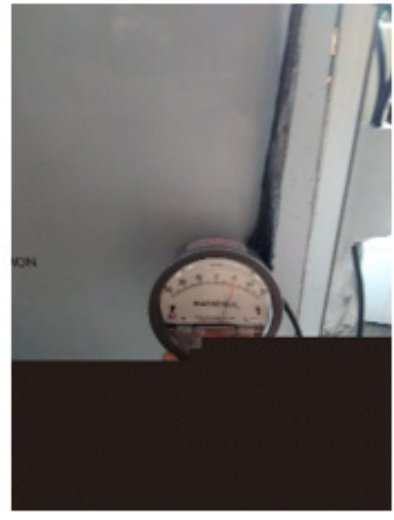

(c)

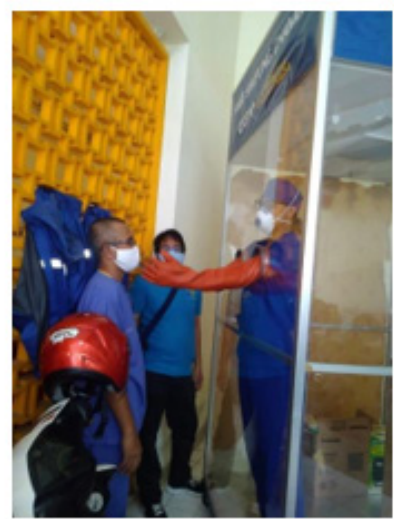

(e)

Figure 3. GAMA Swab Sampling Chamber, (a) prototype, (b) flow simulation, (c) pressure difference measurement, (d) impermeability test and (e) functional test

the screening centers, but the safety of health workers and patients is the top priority. Crosscontamination should also be minimized during oropharyngeal and nasopharyngeal swabs collection process. ${ }^{6}$

The use of a sampling chamber for collecting COVID-19 patient swabs is one of the solutions that has proven to be effective and efficient. The use of a sampling chamber in South Korea has been shown to provide significant benefits because it can do swab sampling quickly and in large quantities, saves the use of PPE and prevents the spread of aerosol contamination due to sampling. ${ }^{7-9} A$ similar sampling chamber was also used at Stanford University Hospital, USA, during the influenza pandemic several years ago. ${ }^{10}$

Gadjah Mada Hospital, as one of the COVID-19 referral hospitals in Yogyakarta, has the capacity of 107 beds intended for caring for patients with COVID-19. Gadjah Mada Hospital also provides a specialized COVID-19 clinic for outpatient services. With an increasing number of suspected and symptomatic individuals to be tested, there is a need for a safe and efficient screening system. COVID-19 services at Gadjah Mada hospitals are supported by a trained swab sampling team consisting of an ear, nose and throat specialist, clinical microbiology residents, and general practitioners. The Gadjah Mada Hospital has provided GAMA Swab Sampling Chamber specifically for asymptomatic and suspected patients as well as voluntary person swab sampling.

The GAMA Swab Sampling Chamber is developed based on the chamber used in the walk-through (WT) screening center in South Korea. This chamber is designed to provide a 


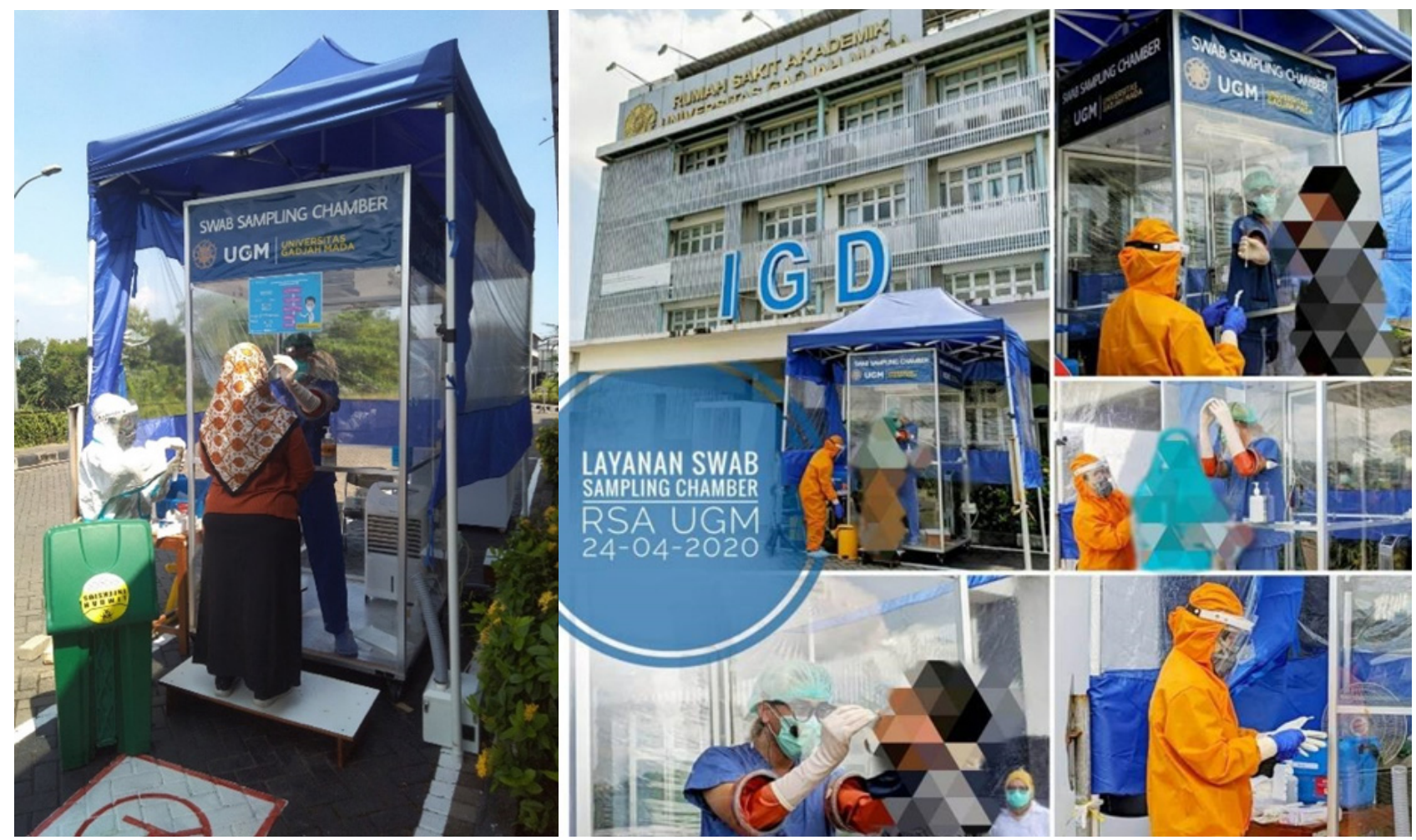

Figure 4. Swab sampling service with a GAMA Swab Sampling Chamber in Gadjah Mada Hospital

Table 1. RT-PCR results for COVID-19 patients whose samples were taken at the GAMA Swab Sampling Chamber

\begin{tabular}{llll}
\hline Groups & $\begin{array}{l}\text { Number of samples } \\
\mathbf{n}\end{array}$ & $\begin{array}{l}\text { Positive result } \\
\mathbf{n}(\%)\end{array}$ & $\begin{array}{l}\text { Negative result } \\
\mathbf{n}(\%)\end{array}$ \\
\hline Asymptomatic patient & 51 & $0(0)$ & $51(100)$ \\
Suspected patient & 72 & $2(2.8)$ & $70(97.2)$ \\
Voluntary person & 284 & $4(1.4)$ & $280(98.6)$ \\
Total & 407 & $6(1.5)$ & $401(98.5)$ \\
\hline
\end{tabular}

rapid screening, have a minimal risk of infection, and reduce excessive use of medical supplies like PPE. The risk of infection may be reduced by limiting the exposure to patients or direct contacts between the patients and healthcare workers. The WT screening center in South Korea used a negative and positive pressure chamber. In the WT screening center using a negative pressure chamber, the patients stayed inside the disinfected chamber, while the healthcare worker is outside the chamber, disallowing a direct contact between the two. In the positive pressure chamber, the health care worker stays inside the chamber while the patient stays outside; thus, direct contact between the two is avoided. This strategy has been successfully applied to the COVID-19 outbreak in South Korea. ${ }^{7}$

The GAMA Swab Sampling Chamber is a positive pressure chamber with the healthcare worker positioned inside with the patient outside the chamber. Collecting a swab with a positive-pressure chamber provides advantages because there is no need to clean the chamber after sampling the patient so that the process can be done faster. The time for swab sampling with this chamber is around $15-20$ minutes. A similar duration of time was reported in the use of a sampling chamber in South Korea. In the WT screening center in South Korea, sampling 
with a negative pressure chamber takes about 20 minutes per patient, while sampling with a positive pressure chamber requires a shorter time, which is 15 minutes per patient. ${ }^{7}$

A swab sampling process is a procedure that potentially produces droplets. Accordingly, it is essential to ensure that droplets do not contaminate the surrounding environment or become a potential for cross-contamination between patients. To overcome this risk, the GAMA Swab Sampling Chamber is equipped with an $\mathrm{H}_{2} \mathrm{O}_{2}$ dry mist disinfectant, which is used to disinfect the surface of the chamber facing the patient. Thus, any droplets attached to the surface of the chamber or gloves can be safely removed. Analysis of 22 related studies showed that human coronaviruses such as SARS coronavirus, Middle East Respiratory Syndrome (MERS) coronavirus, and endemic human coronavirus (HCoV) can survive on the surface of inanimate objects such as metals, glass, plastics for up to 9 days. Nevertheless, these viruses can be inactivated by disinfection of the surface of the object using ethanol $61-71 \%, \mathrm{H}_{2} \mathrm{O}_{2} 0.5 \%$, and Na hypochlorite $0.1 \%$ for 1 minute. ${ }^{11}$

Research showed that exposure to $\mathrm{H}_{2} \mathrm{O}_{2}$ vapor (HPV) created by changing $35 \% \mathrm{w} / \mathrm{w}$ liquid $\mathrm{H}_{2} \mathrm{O}_{2}$ into HPV in several viruses, namely FCV (strain 255) as a representative of human norovirus, Purdue strain of Transmissible Gastroenteritis Virus (TGEV) as representatives of the SARS virus (severe acute respiratory syndrome), human adenovirus type 1 (hADV-1), AIV (A/chicken) Maryland/2007 [H9N9]), and SwIV (A/swine/ Minnesota/2010 [H3N2]) resulted in evidence that HPV can kill the viruses tested. The results of that research supports the use of $\mathrm{H}_{2} \mathrm{O}_{2}$ dry mist in this sampling chamber. ${ }^{12}$

In the sampling process, droplets may be flying in the air. The sampling chamber is placed in open space, so that the exposure of ultraviolet (UV) from the sunlight can inactivate the formed droplets to avoid environmental contamination. Based on the wavelength, UV light can be classified into three bandwidths, UV-A (320-400 $\mathrm{nm})$, UV-B (280-320 nm), and UV-C (200-280 $\mathrm{nm})$. UV-A is the main ultraviolet component of sunlight that reaches the ground. While UV-B only has the effect of killing weak germs, only a small portion of UV-B reaches the earth's surface because most of it is absorbed by the atmosphere. UV rays that are commonly used for disinfection are UV rays whose wavelengths are below $320 \mathrm{~nm}$. Unfortunately, the majority of UV rays that reach the ground are UV-A, with only little UV-B, and very little UV-C. Based on this pattern, it can be said that UV rays that reach the ground are less effective as a germicidal agent. ${ }^{13,14}$ Therefore, the sampling chamber equipped with a UV-C light source, which is turned on every day after the swab removal procedure was added. UV-C is used for the disinfection of viruses and other germs on the surface of the chamber. UV-C is very damaging to cells because their nucleic acids absorb it. Microbes are susceptible to light at wavelengths around $253.7 \mathrm{~nm}$ because the maximum wavelength absorbed by DNA molecules is $260 \mathrm{~nm}$. Also, the effectiveness of UV-C has been proven in viruses in the air carried by aerosols. For example, a very low dose UV ( 2 $\mathrm{mJ} / \mathrm{cm}^{2}$ ) with a 222-nm wavelength deactivates more than $95 \%$ of the H1N1 virus in the air. ${ }^{15,16}$

The use of the sampling chamber is still limited to collecting samples of standing patients and cannot be done on patients lying down. Although limited to standing patients, the use of this sampling chamber provides great benefits because it facilitates the sampling process of patients with COVID-19 so that large numbers of patients can be tested in a short time, thereby reducing the PPE use and the need for a negative pressure isolation room.

\section{Conclusions}

The use of the GAMA Swab Sampling Chamber can simplify the process of collecting swab samples from patients with COVID-19, so that the sampling for a large number of patients can be done in a short time. The use of this chamber also reduces the need for PPE usage and a negative pressure isolation room which are limited in the current pandemic situation. The GAMA Swab Sampling Chamber can be used 
for a safe and efficient swab sampling system, even though it is still limited to patients in the stand-up position.

\section{Acknowledgement}

We would like to thank Universitas Gadjah Mada (UGM) for funding assistance in the COVID-19 pandemic research scheme, Gadjah Mada Hospital which has collaborated in the development of the GAMA Swab Sampling Chamber, eSVe FabLab Vocational School UGM which participated in designing and producing the GAMA Swab Sampling Chamber prototype, Laboratory of Microbiology, Faculty of Medicine, Public Health and Nursing, UGM which conducted RT-PCR testing, and Pusat Kajian dan Pengembangan Kebijakan Industrialisasi (PPKI) UGM which supported this chamber development. We also want to thank dr. Syahra Kurnia Putri for critical editing of the manuscript.

\section{Conflict of interests}

The authors declare that there is no conflict of interest.

\section{References}

1. World Health Organization. WHO DirectorGeneral's opening remarks at the media briefing on COVID-19 - 11 March 2020 [Internet]. WHO Director General's speeches. 2020 [cited 2020 Jun 11]. p. 4. Available from: https://www.who. int/dg/speeches/detail/who-director-generals-opening-remarks-at-the-media-briefing-oncovid-19---11-march-2020

2. Worldometer. Coronavirus Cases [Internet]. Worldometer. 2020 [cited $2020 \mathrm{Jul}$ 2]. Available from: https://www.worldometers.info/ coronavirus/coronavirus-cases/\#daily-cases

3. Kementerian Kesehatan. Infeksi Emerging [Internet]. 2020 [cited 2020 Jul 2]. Available from: https://infeksiemerging.kemkes.go.id/

4. Wang D, Hu B, Hu C, Zhu F, Liu X, Zhang J, et al. Clinical Characteristics of 138 Hospitalized Patients with 2019 Novel Coronavirus-Infected Pneumonia in Wuhan, China. J Am Med Assoc. 2020;323(11):1061-9.

5. High efficiency air filters (EPA, HEPA and ULPA)
Part 1: Classification, performance testing, marking. British Standard. 2009. 1-24 p.

6. Lee J. COVID-19 screening center: how to balance between the speed and safety?. J Korean Med Sci. 2020;35(15).

7. Choi S, Han C, Lee J, Kim S II, Kim IB. Innovative screening tests for covid-19 in south korea. Clin Exp Emerg Med. 2020;7(2):73-7.

8. Kwon KT, Ko JH, Shin H, Sung M, Kim JY. Drivethrough screening center for covid-19: A safe and efficient screening system against massive community outbreak. J Korean Med Sci 2020;35(11).

9. Lee D, Lee J. Testing on the move: South Korea's rapid response to the COVID-19 pandemic. Transportation Research Interdisciplinary Perspectives. 2020;5.

10. Weiss EA, Ngo J, Gilbert GH, Quinn J V. DriveThrough Medicine: A Novel Proposal for Rapid Evaluation of Patients During an Influenza Pandemic. Ann Emerg Med. 2010;55(3):268-73.

11. Kampf G, Todt D, Pfaender S, Steinmann E. Persistence of coronaviruses on inanimate surfaces and their inactivation with biocidal agents. Journal of Hospital Infection. 2020;104(3):246-51.

12. Goyal SM, Chander Y, Yezli S, Otter JA. Evaluating the virucidal efficacy of hydrogen peroxide vapour. J Hosp Infect. 2014;86(4):255-9.

13. Kowalski W. Ultraviolet germicidal irradiation handbook: UVGI for air and surface disinfection. Springer. 2009. 1-501 p.

14. Pozo-Antonio JS, Sanmartín P. Exposure to artificial daylight or UV irradiation (A, B or C) prior to chemical cleaning: an effective combination for removing phototrophs from granite. Biofouling. 2018;34(8):851-69.

15. Tseng CC, Li CS. Inactivation of virus-containing aerosols by ultraviolet germicidal irradiation. Aerosol Sci Tech. 2005;39(12):1136-42.

16. Welch D, Buonanno M, Grilj V, Shuryak I, Crickmore C, Bigelow AW, et al. Far-UVC light: A new tool to control the spread of airbornemediated microbial diseases. Sci Rep. 2018;8(1). 